Supplement of Hydrol. Earth Syst. Sci., 19, 4307-4315, 2015

http://www.hydrol-earth-syst-sci.net/19/4307/2015/

doi:10.5194/hess-19-4307-2015-supplement

(C) Author(s) 2015. CC Attribution 3.0 License.

(c) (i)

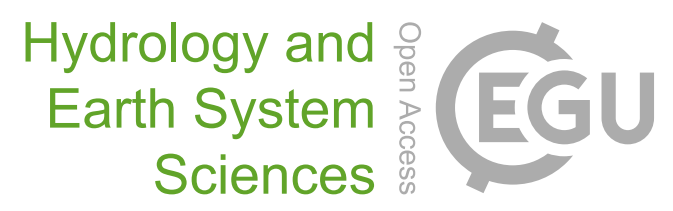

Supplement of

\title{
Historical changes in frequency of extreme floods in Prague
}

\section{Elleder}

Correspondence to: L. Elleder (elleder@chmi.cz)

The copyright of individual parts of the supplement might differ from the CC-BY 3.0 licence. 
1 Estimated maximal water levels and discharges (equal or greater then $\mathrm{Q}_{2}$ ) for

2 pre-instrumental and early instrumental period 1118-1824 (FRPs are in grey, POTQ10 are in $3 \mathrm{red}$ ).

\begin{tabular}{|c|c|c|c|c|c|c|c|c|c|c|c|}
\hline N. & Year & $\mathbf{M}$ & D & $\begin{array}{l}\text { Water level } \\
\text { estimation }\end{array}$ & $\mathbf{E F}$ & $\mathbf{I J}$ & $\begin{array}{l}\text { Hest } \\
{[\mathrm{cm}]}\end{array}$ & $\begin{array}{l}\text { Qest } \\
{\left[\mathrm{m}^{3} \cdot \mathrm{s}^{-1}\right]}\end{array}$ & $\mathbf{A}$ & $\mathbf{G}$ & $\mathbf{C}$ \\
\hline 1 & 1118 & & & $\begin{array}{c}\text { OMF, } \\
\text { Wli: 5m a. Br. }\end{array}$ & $\mathrm{C}$ & & 650 & 6000 & 1 & 100 & B \\
\hline 2 & 1126 & 2 & 22 & Ii: D? & & & 250 & 1800 & 1 & 50 & B \\
\hline 3 & 1141 & 4 & 15 & Ii: D? & & & 300 & 2500 & 1 & 100 & $\mathrm{~B}$ \\
\hline 4 & 1250 & 7 & 15 & F? & & & 250 & 1800 & 1 & 50 & B \\
\hline 5 & 1257 & 8 & 8 & Ii: DH & & & 300 & 2500 & 1 & 75 & B \\
\hline 6 & 1264 & 12 & 7 & Ii: DH & & & 250 & 1800 & 1 & 50 & B \\
\hline 7 & 1270 & $?$ & $?$ & $F !$ & & & 200 & 1500 & 1 & 50 & B \\
\hline 8 & 1272 & 3 & 19 & Ii: DBr & & $\mathrm{I} ?$ & 420 & 3500 & 2 & 100 & B \\
\hline 9 & 1273 & 8 & 25 & WLi: Ni & & & 420 & 3500 & 1 & 100 & B \\
\hline 10 & 1281 & 4 & 1 & Ii: MO & & & 300 & 2500 & 1 & 50 & B \\
\hline 11 & 1315 & 8 & 2 & Ii: DM & & & 300 & 2500 & 1 & 50 & B \\
\hline 12 & 1316 & $?$ & $?$ & Ii: DM & $\mathbf{C}$ & & 300 & 2500 & 1 & 50 & B \\
\hline 13 & 1321 & 7 & 4 & Ii: DH & & & 300 & 2500 & 1 & 50 & B \\
\hline 14 & 1322 & $?$ & $?$ & DiB: BE & & & 250 & 1800 & 1 & 90 & B \\
\hline 15 & 1327 & 6 & 0 & Ii: Agr & & & 200 & 1500 & 1 & 100 & B \\
\hline 16 & 1342 & 8 & 2 & Ii: DBr, DW, DM & & I? & 420 & 3500 & 1 & 100 & B \\
\hline 17 & 1359 & 9 & 1 & WLi: Ni & $\mathrm{C}$ & & 420 & 3500 & 1 & 100 & B \\
\hline 18 & 1364 & 1 & 27 & $\mathbf{F} !$ & & & 300 & 2500 & 2 & 100 & B \\
\hline 19 & 1367 & 11 & 3 & WLi: Ag, Ni & C? & & 440 & 3500 & 1 & 100 & B \\
\hline 20 & 1370 & 8 & 23 & WLi: Ag, Li & & & 420 & 3500 & 1 & 100 & B \\
\hline 21 & 1373 & 3 & 21 & WLi: OTi & & & 420 & 3500 & 2 & 100 & B \\
\hline 22 & 1374 & 2 & 12 & WLi: Ag, Ni & C & & 420 & 3500 & 2 & 100 & B \\
\hline 23 & 1387 & 9 & 20 & WLi: ML & & & 300 & 2500 & 3 & 100 & B \\
\hline
\end{tabular}




\begin{tabular}{|c|c|c|c|c|c|c|c|c|c|c|c|}
\hline 24 & 1392 & 12 & 13 & Ii: DM & & & 300 & 2500 & 1 & 100 & B \\
\hline 25 & 1405 & 7 & 4 & F? & & & 250 & 1900 & 1 & 50 & B \\
\hline 26 & 1432 & 3 & 13 & WLi: Li & & & 420 & 3500 & 1 & 100 & B \\
\hline 27 & 1432 & 7 & 30 & $\begin{array}{l}\text { WLi: Ag, Ni, OTS } \\
\text { Ii: DBr, DH, DW }\end{array}$ & $\mathrm{C}$ & & 650 & 6000 & 1 & 100 & A \\
\hline 28 & 1432 & 12 & 15 & $=1432 \mathrm{III}$ & & & 420 & 3500 & 1 & 100 & B \\
\hline 29 & 1434 & 8 & 5 & DiB: BE & & & 250 & 1800 & 1 & 90 & $\mathrm{C}$ \\
\hline 30 & 1445 & 7 & 3 & WLi: Rcoll & & & 355 & 2700 & 1 & 75 & B \\
\hline 31 & 1464 & 3 & 13 & Ii: FO & & & 250 & 1900 & 3 & 50 & $\mathrm{D}$ \\
\hline 32 & 1481 & 6 & 2 & BM, nose & & & 355 & 2700 & 1 & 100 & B \\
\hline 33 & 1481 & 6 & 17 & BM, pate & & & 395 & $\begin{array}{r}3000 \\
\mathbf{3 1 0 0} \\
\end{array}$ & 1 & 100 & A \\
\hline 34 & 1501 & 8 & 25 & $\begin{array}{c}\text { BM, } 120 \mathrm{~cm} \text { above } \\
\text { a head } \\
\text { Ii: Bar } \\
\text { Wli: } \text { ML }\end{array}$ & & & 516 & $\begin{array}{l}4000 \\
\underline{4200} \\
\end{array}$ & 1 & 100 & A \\
\hline 35 & 1504 & 3 & 16 & Ii: D? & & & 200 & 1500 & 1 & 100 & A \\
\hline 36 & 1511 & 7 & 31 & DiB: UV & & & 200 & 1500 & 1 & 75 & \\
\hline 37 & 1515 & 7 & 3 & Ii: MO & & & 300 & 2200 & 1 & 100 & A-B \\
\hline 38 & 1515 & 7 & 31 & BM, beard & $\mathrm{C}$ & & 335 & $\begin{array}{l}2600 \\
\underline{\mathbf{2 6 0 0}} \\
\end{array}$ & 1 & 100 & A-B \\
\hline 39 & 1515 & 9 & 3 & F? & & & 200 & 1500 & 1 & 100 & $\mathrm{C}$ \\
\hline 40 & 1521 & 6 & 5 & Ii: MO & & & 250 & 1800 & 1 & 100 & B \\
\hline 41 & 1523 & 7 & 14 & Ii: MO & & & 200 & 1500 & 1 & 100 & B \\
\hline 42 & 1523 & 11 & 20 & Ii: WT & & $\mathrm{I} ?$ & 240 & 1800 & 1 & 100 & \\
\hline 43 & 1528 & 6 & 26 & Ii: WT & $\mathbf{C}$ & & 250 & 1900 & 1 & 100 & B \\
\hline 44 & 1531 & 5 & 11 & BM & & & 370 & $\begin{array}{l}2800 \\
\underline{\mathbf{2 9 0 0}}\end{array}$ & 1 & 100 & B \\
\hline 45 & 1533 & $?$ & $?$ & F? & & & 200 & 1500 & 1 & 100 & $\mathrm{C}$ \\
\hline
\end{tabular}




\begin{tabular}{|c|c|c|c|c|c|c|c|c|c|c|}
\hline 46 & 1534 & $?$ & $?$ & DiB: BE & & 200 & 1500 & 1 & 75 & $\mathrm{C}$ \\
\hline 47 & 1537 & 6 & 2 & BM: eyes, Ii: WT & $\mathbf{C}$ & 375 & 2900 & 1 & 100 & A \\
\hline 48 & 1544 & 9 & 1 & Ii: D?, WLi: KK & $\mathbf{C}$ & 250 & 1900 & 1 & 100 & $\mathrm{~B}$ \\
\hline 49 & 1545 & 7 & 24 & Ii: D? & & 250 & 1900 & 2 & 100 & $\mathrm{~B}$ \\
\hline 50 & 1554 & 1 & & DiB: OT & & 200 & 1500 & 3 & 75 & $\mathrm{~B}$ \\
\hline 51 & 1560 & 12 & 15 & Wli: Cell & & 250 & 1900 & 2 & 100 & $\mathrm{~B}$ \\
\hline 52 & 1563 & 6 & 11 & Ii: D? & $\mathbf{C}$ & 200 & 1500 & 1 & 90 & $\mathrm{~B}$ \\
\hline 53 & 1564 & 7 & 14 & $\begin{array}{c}\text { BM, beard } \\
\text { Wli: Rcoll, Acoll }\end{array}$ & & 335 & 2600 & 2 & 100 & B \\
\hline 54 & 1566 & 2 & 15 & BM, mouth & & 345 & 2700 & 3 & 100 & A \\
\hline 55 & 1567 & 9 & 10 & BM, below a head & $\mathbf{C}$ & 335 & 2600 & 3 & 100 & B \\
\hline 56 & 1568 & 6 & 23 & BM, eyebrow & & 380 & 3000 & 1 & 100 & B \\
\hline 57 & 1569 & 7 & 1 & BM, eyes & C & 375 & $\begin{array}{l}2900 \\
\underline{\mathbf{2 9 0 0}} \\
\end{array}$ & 1 & 100 & $\begin{array}{l}\text { A- } \\
\text { B }\end{array}$ \\
\hline 58 & 1570 & 2 & 10 & BM, mouth & & 350 & 2700 & 1 & 100 & A \\
\hline 59 & 1575 & $?$ & $?$ & BM, beard & & 335 & 2600 & 2 & 100 & B \\
\hline 60 & 1582 & 5 & 12 & BM, eyes & & 370 & 2800 & 1 & 100 & A \\
\hline 61 & 1582 & 6 & 13 & $\begin{array}{c}\text { BM, eyebrow } \\
\text { Ii: Bar }\end{array}$ & & 385 & $\begin{array}{l}3000 \\
\underline{\mathbf{3 0 0 0}} \\
\end{array}$ & 1 & 100 & A \\
\hline 62 & 1587 & 6 & 8 & BM, eyes & & 375 & 2900 & 1 & 100 & B \\
\hline 63 & 1595 & 3 & 29 & DiB: UV, LU, EL & $\mathbf{C}$ & 200 & 1500 & 1 & 75 & B \\
\hline 64 & 1595 & 8 & 17 & F? & & 250 & 1800 & 1 & 100 & \\
\hline 65 & 1598 & 3 & 17 & $\begin{array}{c}\text { BM, } 90 \mathrm{~cm} \text { above } \\
\text { a head }\end{array}$ & & 485 & $\begin{array}{l}3700 \\
\mathbf{4 0 0 0} \\
\end{array}$ & 1 & 100 & A \\
\hline 66 & 1598 & 8 & 18 & $\begin{array}{c}\text { BM, } 90 \mathrm{~cm} \text { above } \\
\text { a head }\end{array}$ & $\mathbf{C}$ & 485 & $\begin{array}{l}3800 \\
\underline{\mathbf{4 0 0 0}} \\
\end{array}$ & 1 & 100 & A \\
\hline 67 & 1604 & 12 & 15 & Ii: D? & & 200 & 1500 & 3 & 100 & $\mathrm{~B}$ \\
\hline 68 & 1613 & 6 & 14 & Ii: D? & & 200 & 1500 & 1 & 100 & B \\
\hline
\end{tabular}




\begin{tabular}{|c|c|c|c|c|c|c|c|c|c|c|c|}
\hline 69 & 1615 & 3 & 18 & BM, above a head & & & 405 & 3100 & 1 & 100 & A \\
\hline 70 & 1629 & 10 & 1 & $\begin{array}{l}\text { Wli: Val, Li, } \\
\text { Ii: MO, } \\
\text { DiB: EL }\end{array}$ & & & 350 & 2400 & 2 & 100 & A \\
\hline 71 & 1651 & 1 & 18 & DiB: UV, EL & $\mathbf{W}$ & & 290 & 2400 & 1 & 75 & B \\
\hline 72 & 1651 & 5 & 11 & Ii: WT, D? & & & 300 & 2500 & 2 & 100 & B \\
\hline 73 & 1651 & 12 & 2 & Ii: WT, D! & & & 300 & 2500 & 2 & 100 & B \\
\hline 74 & 1655 & 2 & 14 & Ii: D?, DiB: EL & $\mathbf{C}$ & & 500 & 4000 & 1 & 100 & A \\
\hline 75 & 1656 & 6 & 12 & DiB: UV, LU & & & 200 & 1500 & 1 & 75 & $\mathrm{C}$ \\
\hline 76 & 1658 & & & DiB: EL & $\mathbf{W}$ & & 310 & 2400 & $?$ & 50 & $\mathrm{C}$ \\
\hline 77 & 1668 & 3 & 21 & DiB: UV & & & 200 & 1500 & 1 & 100 & B \\
\hline 78 & 1670 & 3 & 15 & DiB: BE, EL & & & 270 & 2200 & 1 & 75 & $\mathrm{C}$ \\
\hline 79 & 1675 & 6 & 23 & FM, Wli: Ag & & & 485 & $\begin{array}{l}3800 \\
\underline{\mathbf{3 9 0 0}} \\
\end{array}$ & 1 & 100 & A \\
\hline 80 & 1682 & 1 & 26 & Wli: Huk & $\mathbf{C}$ & & 420 & 3500 & 1 & 100 & $\mathrm{~B}$ \\
\hline 81 & 1698 & 7 & 23 & Wli: SIs & & & 350 & 2800 & 1 & 100 & B \\
\hline 82 & 1712 & 4 & 24 & FM & & & 368 & 3000 & 1 & 100 & A \\
\hline 83 & 1714 & 7 & 31 & $\begin{array}{c}\text { DiB: SA } \\
\text { Ii: WT, ST }\end{array}$ & & & 250 & 1800 & 1 & 100 & $\mathrm{C}$ \\
\hline 84 & 1734 & 6 & 21 & $\begin{array}{c}\text { FM* } \\
=1740{ }_{\mathrm{XII}}-60 \mathrm{~cm}\end{array}$ & & & 265 & 2000 & 1 & 100 & A \\
\hline 85 & 1736 & 6 & 19 & $\begin{array}{c}\text { FM, BM, nose } \\
\text { WLi: ML }\end{array}$ & & & 369 & 3000 & 1 & 100 & A \\
\hline 86 & 1740 & 3 & 16 & DiB: OT, Ii: D? & $\mathbf{W}$ & $\mathrm{I} ?$ & 200 & 1500 & 1 & 100 & A \\
\hline 87 & 1740 & 8 & 25 & Ii: DW & & & 130 & 1300 & 1 & 100 & B \\
\hline 88 & 1740 & 12 & 22 & $\mathbf{F M} *$ & & & 325 & 2500 & 1 & 100 & B \\
\hline 89 & 1741 & 1 & 13 & F? & & & 200 & 1500 & 1 & 100 & A \\
\hline 90 & 1741 & 2 & 4 & $=1734$ & & & 265 & 2000 & 1 & 100 & B \\
\hline
\end{tabular}




\begin{tabular}{|c|c|c|c|c|c|c|c|c|c|c|c|}
\hline 91 & 1745 & 6 & 15 & Wli: FO, WT & & & 230 & 1800 & 1 & 100 & B \\
\hline 92 & 1746 & 4 & 23 & Wli: WT & & & 200 & 1500 & 1 & 100 & B \\
\hline 93 & 1747 & 4 & 2 & F? & & & 265 & 2000 & 1 & 100 & A \\
\hline 94 & 1747 & 12 & 14 & $>1734$ & & & 270 & 2100 & 1 & 100 & B \\
\hline 95 & 1750 & 6 & 17 & FM, $=1734$ & & & 265 & 2000 & 1 & 100 & A \\
\hline 96 & 1750 & 7 & 12 & $\mathrm{FM}^{*},>1740$ & & & 295 & 2200 & 1 & 100 & A \\
\hline 97 & 1752 & 8 & 13 & $\mathrm{FM}^{*},=1734$ & & & 265 & 2000 & 1 & 100 & A \\
\hline 98 & 1755 & 7 & 7 & $\mathrm{FM}^{*},=1750_{\mathrm{VII}}$ & & & 295 & 2200 & 1 & 100 & A \\
\hline 99 & 1757 & 5 & 31 & Ii:D?, WLi: OTl & & & 280 & 2100 & 1 & 100 & B \\
\hline 100 & 1769 & 12 & 21 & DiB: BE & & $\mathrm{I} ?$ & 270 & 2000 & 1 & 75 & $\mathrm{C}$ \\
\hline 101 & 1770 & 4 & 4 & FM* & & & 295 & 2500 & 1 & 100 & A \\
\hline 102 & 1771 & 3 & 17 & FM & & & 415 & 3200 & 1 & 100 & A \\
\hline 103 & 1775 & 2 & 5 & DiB: BE & & & 250 & 2000 & 1 & 75 & A \\
\hline 104 & 1777 & 2 & 25 & DiB: BE & & & 200 & 1500 & 1 & 75 & A \\
\hline 105 & 1782 & 3 & 23 & EM & & & 297 & 2300 & 1 & 100 & A \\
\hline 106 & 1784 & 2 & 28 & EM & W & & 575 & 4400 & 1 & 75 & A \\
\hline 107 & 1785 & 4 & 16 & EM & $\mathbf{C}$ & & 280 & 2100 & 1 & 100 & A \\
\hline 108 & 1786 & 8 & 17 & EM & & & 336 & 2500 & 1 & 100 & A \\
\hline 109 & 1796 & 12 & 24 & EM & & & 267 & 2100 & 1 & 100 & A \\
\hline 110 & 1799 & 1 & 22 & EM & W & & & 3900 & 1 & 100 & A \\
\hline 111 & 1803 & 2 & 19 & EM & & & 238 & 1800 & 1 & 100 & A \\
\hline 112 & 1803 & 3 & 3 & EM & & & 208 & 1600 & 1 & 100 & A \\
\hline 113 & 1804 & 6 & 15 & EM & $\mathbf{C}$ & & 354 & 2700 & 1 & 100 & A \\
\hline 114 & 1805 & 2 & 26 & EM & & & 267 & 2100 & 1 & 100 & A \\
\hline 115 & 1807 & 2 & 14 & EM & & & 238 & 1830 & 1 & 100 & A \\
\hline 116 & 1809 & 1 & 28 & EM & & & 320 & 2450 & 1 & 100 & A \\
\hline 117 & 1810 & 3 & 3 & EM & & & 312 & 2400 & 1 & 100 & A \\
\hline 118 & 1814 & 3 & 23 & EM & & & 207 & 1600 & 3 & 75 & A \\
\hline
\end{tabular}




\begin{tabular}{c|c|c|c|c|c|c|c|c|c|c|c}
\hline 119 & $\mathbf{1 8 1 5}$ & $\mathbf{8}$ & 9 & $\mathbf{E M}$ & & & 267 & 2060 & 1 & 75 & A \\
\hline 120 & $\mathbf{1 8 2 0}$ & $\mathbf{1}$ & 20 & $\mathbf{E M}$ & & & 208 & 1600 & 1 & 100 & A \\
\hline 121 & $\mathbf{1 8 2 1}$ & $\mathbf{3}$ & 9 & $\mathbf{E M}$ & & & 191 & 1500 & 3 & 100 & A \\
\hline 122 & $\mathbf{1 8 2 4}$ & $\mathbf{6}$ & 26 & $\mathbf{E M}$ & & & 444 & 3760 & 1 & 100 & A \\
\hline
\end{tabular}

\section{Explanatory notes:}

- Year, M (month), D (day)

The dates before 1584 were recalculated according to Gregorian calendar

\section{- Water level and discharge estimation approach Ii (Impact indicator):}

$\mathrm{D}$ (damages) $+(\mathrm{H}$ - houses, $\mathrm{M}$ - mills, $\mathrm{W}$ - weirs, $\mathrm{Br}$ - bridge, ? - in general, ! - big damages, Bar - barriers in front of the bridge, Agr: damages in agriculture)

$$
\text { FO - Ferries stopped }(>120 \mathrm{~cm})
$$

WT - Wood taken from lumber yards away $(>250 \mathrm{~cm})$

ST - Salt warehouses flooded and a supplies taken away $(>250 \mathrm{~cm})$

MO - Mills out of order $(250-300 \mathrm{~cm})$

Wli (water level indictor): the important buildings and squares,

Acoll: Angel College of Prague University, Ag: St. Giles, Cell: cellars in general, Huk: House "U kř́̌žku", Li: St. Leonhard, KK: Kings Kiln, ML: St. Mary in Lacu, Ni: St. Nicholas, OTi: important part of Old Town, OTl: lower part of Old Town, OTS: Old Town Square, Rcoll: Reček college of Prague University, SIs: Střelecký island, Val:

St. Valentine.

$5 \mathrm{~m}$ a. Br: $5 \mathrm{~m}$ above wooden bridge

\section{Measured water levels:}

BM - Bearded Man, beard, mouth, nose, eyes, eyebrow, pate (see the picture by Elleder et al (2013)

FM - flood marks, * recently disappeared flood mark

EM - early measurement by Prague Klementinum observatory (Elleder, 2010b)

\section{Dib (Discharge balance):}

UV (the Upper Vltava River), LU (the Lužnice River), OT: (the Otava River), SA (the Sázava River), BE (the Berounka River), EL (the Elbe River)

Other possibilities: F? (Flood in general), BF (Big flood), OMF (Flood out of memory) Underlining: dominant indicator 
3 Hest: $\mathrm{H}[\mathrm{cm}]$ water level estimation

Qest: $\mathrm{Q}\left[\mathrm{m}^{3} . \mathrm{s}^{-1}\right]$ peak discharge estimation, underlined values: hydraulic approach (Elleder et al, 2013).

IJ: Ice jam, possible ice jam impact for some water levels $<300 \mathrm{~cm}$ or floods before the year 1350 .

EF: European floods C: CEF (Central Europe - the Elbe, the Oder, the Danube, the Rhine), W: WCEF (West and Central - the Elbe, the Oder, the Danube, the Rhine the Loire, the Garrone, the Seine)

A - Authenticity level (1 - based on primary source (eye-witnessed), 2 - documentary source: 50-150 years after the flood, 3 - secondary source: more than 150 years after the flood)

G [\%] - probability of the flood in Prague $100 \%$ - for sure, $90 \%$ - very probable, $75 \%$ probable, $50 \%$ - cannot be excluded

Reliability control, A - flood is recorded in Prague and Dresden, B - in Prague, C - Prague flood assumed 